\title{
Tunnel Geological Prediction Radar Alternating Electromagnetic Field Propagation Attenuation in Lossy Inhomogeneous Medium
}

\author{
Si Yang Chen ${ }^{1, a}$, Yan Peng Zhu ${ }^{1, b}$, Zhong Li ${ }^{1, c}$, Tian Yu Zhang ${ }^{2}$ \\ ${ }^{1}$ Collage of civil engineering,Lanzhou University of Technology,lanzhou730050,China; \\ ${ }^{2}$ Lanzhou military political department management office,lanzhou730000,China;

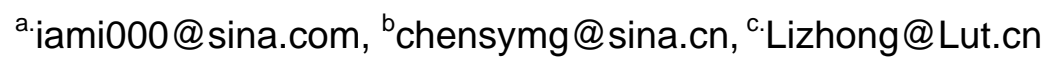

Keywords: Tunnel geological prediction; Radar; Dielectric relaxation properties; Attenuation

\begin{abstract}
Tunnel geological prediction of ground-penetrating radar waves propagation in a lossy inhomogeneous medium, there must be a high-frequency attenuation. We must consider the electrical energy and magnetic energy of the relaxation loss, while the tunnel surrounding the quality factor in determining the attenuation of the necessary elements. Three parameters are not independent of unrelated, but from a different side to reflect the attenuation. Considering the coupling effect between three parameters, culminating in the establishment of the radar electromagnetic waves attenuation coefficient, in inhomogeneous rock medium exists the presence of relaxation phenomenon. For the geological radar effectively identify the reflected wave with different properties, different absorption and attenuation characteristics to analyze and study the surrounding rock structures, are well ahead of geological forecast guidance tunneling significance.
\end{abstract}

\section{Introduction}

Electromagnetic induction method is the important branch of electrical prospecting, using mainly the electrical conductivity, permeability and dielectric properties of geomaterials applied the principle of electromagnetic induction, observation and study of artificial or natural electromagnetic field frequency distribution and time characteristics ${ }^{[1,2]}$, and then solve the various geological problems. GPR is by transmitting and receiving high frequency, narrow pulse wide frequency electromagnetic pulses $(10 \mathrm{MHz}-1 \mathrm{GHz})$, use of different dielectric constant of the medium at the interface will produce electromagnetic wave reflection and the amplitude of the received electromagnetic waves, waveform, frequency characteristics to identify and infer the subsurface structure, stratigraphic and lithologic characteristics of a geophysical technology, with high observational accuracy of the information-rich, the very wide range of applications in the tunnel geological prediction.

Tunnel geological prediction of ground-penetrating radar electromagnetic wave propagation in a lossy inhomogeneous medium, so there must be a high-frequency attenuation. Electromagnetic wave attenuation phenomenon in lossy inhomogeneous media communication process, generally considered to be caused due to electrical conductivity, dielectric relaxation nature and magnetic relaxation properties of a variety of factors ${ }^{[3,4]}$, the theoretical study of the electromagnetic method in the previous discussion alternating the magnetization of the magnetic body in the electromagnetic field in the quasi-steady state conditions, without considering the magnetization state to stabilize the process, while in the electric field changes similar. Power attenuation and magnetic energy decay mechanism is similar.

Basic theory of radar waves underground alternating electromagnetic field propagation in the homogeneous medium

The basic theory of electromagnetic wave propagation. High frequency electromagnetic wave propagation in the medium of obedience to the differential form of Maxwell equations: 


$$
\left\{\begin{array}{l}
\nabla \times H=J+\frac{\partial D}{\partial t} \\
\nabla \times E=-\frac{\partial B}{\partial t} \\
\nabla \bullet B=0 \\
\nabla \bullet D=q
\end{array}\right.
$$

Where, $\mathrm{E}$ is the electric field, $\mathrm{H}$ is the magnetic field strength, $\mathrm{D}$ is the potential vector, $\mathrm{B}$ the magnetic induction, $\mathrm{t}$ is time, $\mathrm{J}$ is current density, $\mathrm{q}$ is the density of free charge.At the same time, there is the following relationship:

$$
\left\{\begin{array}{l}
J=\sigma E \\
D=\varepsilon E \\
B=\mu H
\end{array}\right.
$$

Where $\sigma$ is the conductivity, $\mu$ is permeability, $\varepsilon$ for the dielectric constant. In the conductivity is not zero with media, the bulk charge can not be gathered in one place, so in the electric prospecting in conductive medium $\nabla \bullet D=0$.Maxwell equations in five variables elimination into three, the following equations:

$$
\left\{\begin{array}{l}
\nabla \times H=\sigma E+\varepsilon \frac{\partial E}{\partial t} \\
\nabla \times E=-\mu \frac{\partial H}{\partial t} \\
\nabla \bullet H=0 \\
\nabla \bullet E=0
\end{array}\right.
$$

The equations of the first type of rotation on both sides, and the type second substitution:

$$
\nabla \times \nabla \times H=-\sigma \mu \frac{\partial H}{\partial t}-\varepsilon \mu \frac{\partial^{2} H}{\partial t^{2}}
$$

Because of the vector identity:

$$
\begin{aligned}
& \nabla \times \nabla \times H=\nabla \nabla \bullet H-\nabla^{2} H \\
& \nabla^{2} H=\sigma \mu \frac{\partial H}{\partial t}+\varepsilon \mu \frac{\partial^{2} H}{\partial t^{2}} \\
& \nabla^{2} E=\sigma \mu \frac{\partial E}{\partial t}+\varepsilon \mu \frac{\partial^{2} E}{\partial t^{2}}
\end{aligned}
$$

If the electromagnetic field frequency is high, medium high resistance ( $\sigma \cong 0$ ) can be seen Eq.6, Eq.7 the right end of the first can be ignored following expression:

$$
\begin{aligned}
& \nabla^{2} H=\varepsilon \mu \frac{\partial^{2} H}{\partial t^{2}} \\
& \nabla^{2} E=\varepsilon \mu \frac{\partial^{2} E}{\partial t^{2}}
\end{aligned}
$$

Because $v=1 / \sqrt{\varepsilon \mu}$, so we can get:

$$
\left\{\begin{array}{l}
\nabla^{2} E=v^{-2} \frac{\partial^{2} E}{\partial t^{2}} \\
\nabla^{2} H=v^{-2} \frac{\partial^{2} H}{\partial t^{2}}
\end{array}\right.
$$

This shows that electromagnetic field vector $\mathrm{E}, \mathrm{H}$ in free space to speed according to the form of wave speed, size and medium concerned.

Harmonic varying field case of radar electromagnetic waves in a homogeneous medium propagation characteristics. Plural forms of expression of the harmonic varying field as follows:

$$
\left\{\begin{array}{l}
H=H_{0} e^{-i \omega p} \\
E=E_{0} e^{-i \omega p}
\end{array}\right.
$$

Where: $\rho$ resistivity. The Eq.2 into Eq.5, the transformation can be the plural form of the Maxwell equations: 


$$
\left\{\begin{array}{l}
\nabla \times H=-i \omega \varepsilon^{*} E \\
\nabla \times E=i \omega \mu H \\
\nabla \bullet H=0 \\
\nabla \bullet E=0
\end{array}\right.
$$

Where: $\varepsilon^{*}=\varepsilon+i \frac{1}{\omega \mu}$ the complex permittivity.Assume that the electromagnetic wave propagation coefficient is $k=\frac{\omega}{v}=\omega \sqrt{\varepsilon \mu}$.If $\varepsilon$ will change for the plural form of the $\varepsilon^{*}$ propagation coefficient $k=\left(\varepsilon \mu \omega^{2}-i \omega \sigma \mu\right)^{\frac{1}{2}}$, by inference harmonic electromagnetic field differential equations - Helmholtz homogeneous equation:

$$
\left\{\begin{array}{l}
\nabla^{2} H=k^{2} H \\
\nabla^{2} E=k^{2} E
\end{array}\right.
$$

In a uniform medium of resistivity $\mathrm{A}$, make the following assumptions:

1) $\mathrm{X}, \mathrm{Y}$ axis in the wave polarization plane;

2) Z-axis direction of wave propagation;

3) The amplitude of the electromagnetic field in the XOY plane.

Then caused by the current source vector potential $A$, the formula $A$ desirable $A_{x}, A_{y}, A_{z}$, the existence:

$$
\frac{\partial^{2} A}{\partial z^{2}}=k_{1}^{2} A
$$

Solving Eq.14, can be obtained:

$$
A=C_{1} e^{-k_{1} z}+C_{2} e^{k_{1} z}
$$

The first positive wave, gradually reduced from the electromagnetic radiation source, the second negative wave, the medium is homogeneous medium negative wave $C_{2}=0$, the final solution:

$$
A_{x, y, z}=C_{x, y, z} e^{-k_{1} z}
$$

The component of the electromagnetic field solution:

$$
\left\{\begin{array}{l}
H_{x}=C_{y} k_{1} e^{-k_{,},}, H y=-C_{x} k_{1} e^{-k_{2} z}, H_{z}=0 \\
E_{x}=C_{x} i \omega e^{-k_{1},}, E y=-C_{y} i \omega \mu e^{-k_{1},}, E_{z}=0
\end{array}\right.
$$

The above kinds of plural forms, and attenuation along the Z-axis direction, if the propagation coefficient $k_{1}$ is divided into real and imaginary part, assume that:

$k_{1}=b+a i$

Combination of wave propagation coefficient Eq.14, the square after the solution:

$$
\left\{\begin{array}{l}
b=\omega \sqrt{\varepsilon \mu} \sqrt{\frac{1}{2}\left(\sqrt{1+m^{2}}-1\right)} \\
a=-\omega \sqrt{\varepsilon \mu} \sqrt{\frac{1}{2}\left(\sqrt{1+m^{2}}+1\right)}
\end{array}\right.
$$

Where $m=\frac{\sigma}{\omega \varepsilon}$ is the electromagnetic coefficient of the medium, Eq.19 into Eq.18,available to the Ex component:

$$
E_{x}=\left|E_{x}\right| e^{-i \omega x}=\omega \mu C_{x} e^{-b z} e^{-i\left(\omega x+a z-\frac{\pi}{2}\right)}
$$

Amplitude and phase can be drawn from the above equation:

$$
\begin{aligned}
& \left|E_{x}\right|=\omega \mu C_{x} e^{-b z} \\
& \varphi(t)=\omega t+a z-\frac{\pi}{2}
\end{aligned}
$$

Can be called $a$ phase coefficient, attenuation coefficient for the $b$.The above-mentioned regularity in physics can be interpreted as: when the propagation of the electromagnetic field in a conductor, the induced current, resulting in heat loss, the better the conductivity of the conductor, the free charge in the medium is more energy consumption. 


\section{Radar electromagnetic wave propagation in inhomogeneous media loss mechanism}

Dielectric relaxation and magnetic relaxation of dissipative effects in some sense, but so far, for this type of dissipation effect is still weak links ${ }^{[5]}$.Due to the non-uniform medium of dielectric relaxation and magnetic relaxation is the major factor in the attenuation of the rock in the media is a non-homogeneous medium, the propagation of the geological radar in the wall rock.

Radar electromagnetic waves in inhomogeneous medium the loss of electric energy. The introduction of the complex dielectric constant, the non uniform medium become relaxation polarization medium:

$$
\begin{aligned}
& \mathcal{E}^{*}=\varepsilon_{\infty}+\frac{\mathcal{E}_{l}-\varepsilon_{\infty}}{1+i \omega \tau} \\
& \left\{\begin{array}{l}
\mathcal{E}^{*}=\varepsilon^{\prime}-\mathcal{E}^{\prime \prime} i \\
\operatorname{tg} \theta=\frac{\varepsilon^{\prime \prime}}{\mathcal{E}^{\prime}}
\end{array}\right.
\end{aligned}
$$

Where: $\varepsilon^{\prime}$ for the complex permittivity of the real part of the dielectric constant, that is the usual dielectric constant; $\varepsilon^{\prime \prime}$ is the imaginary part, also called the loss factor, $i=\sqrt{-1}, \varepsilon_{\infty}$ and $\varepsilon_{l}$ were measured in conditions of high and low frequency relative permittivity. $\frac{\rho}{\omega \varepsilon_{0}}$ can ignore the case of high frequency electromagnetic wave, $\tau$ is relaxation time. $\varepsilon^{\prime}$ and $\varepsilon^{\prime \prime}$ and the frequency of the relaxation polarization medium can be expressed as:

$$
\left\{\begin{array}{l}
\varepsilon^{\prime}=\varepsilon_{\infty}+\frac{\varepsilon_{l}-\varepsilon_{\infty}}{1+(\omega \tau)^{2}} \\
\mathcal{E}^{\prime \prime}=\frac{\left(\varepsilon_{l}-\varepsilon_{\infty}\right) \omega \tau}{1+(\omega \tau)^{2}}
\end{array}\right.
$$

In the case of a single relaxation, from Eq.25 to eliminate $\omega t$, cole-cole model can be a form of expression:

$$
\left(\varepsilon^{\prime}-\varepsilon_{0}^{\prime}\right)^{2}+\left(\varepsilon^{\prime \prime}\right)^{2}=R^{2}
$$

In the case of a group of relaxation time ,KS Carl and RH Carr 's study pointed out that the relationship between $\varepsilon^{\prime}$ and $\varepsilon^{\prime \prime}$ is still a circle. $\varepsilon^{*}$ frequency relations from Eq.23 becomes:

$$
\varepsilon^{*}=\varepsilon_{\infty}+\frac{\varepsilon_{l}-\varepsilon_{\infty}}{1+(i \omega \tau)^{1-\alpha}}
$$

Where $0<\alpha<1$ represents the dispersion of the relaxation time. Eq.26 be expressed as a negative form:

$$
\left\{\begin{array}{l}
\varepsilon^{\prime}=\varepsilon_{\infty}+\left(\varepsilon_{l}-\varepsilon_{\infty}\right) \frac{1+(\omega \tau)^{1-\alpha} \sin \frac{\pi \alpha}{2}}{1+2(\omega \tau)^{1-\alpha} \sin \frac{\pi \alpha}{2}+(\omega \tau)^{2(1-\alpha)}} \\
\varepsilon^{\prime \prime}=\left(\varepsilon_{l}-\varepsilon_{\infty}\right) \frac{(\omega \tau)^{1-\alpha} \cos \frac{\pi \alpha}{2}}{1+2(\omega \tau)^{1-\alpha} \sin \frac{\pi \alpha}{2}+(\omega \tau)^{2(1-\alpha)}}
\end{array}\right.
$$

At this time of electromagnetic waves in an inhomogeneous medium energy loss factor $\varepsilon^{\prime \prime}$ can be solved.

Radar electromagnetic wave in inhomogeneous medium the loss of magnetic energy. Similarly, Electromagnetic wave electrical equations can be transformed to the magnetic equation, when assuming that the permeability of the magnetic body to use the plural to describe:

$$
\mu^{*}=\mu_{\infty}+\frac{\mu_{1}-\mu_{\infty}}{1+(i \omega \tau)^{1-\alpha}}
$$

After a complex operation, the real and imaginary components: 


$$
\left\{\begin{array}{l}
\mu^{\prime}=\mu_{\infty}+\left(\mu_{l}-\mu_{\infty}\right) \frac{1+(\omega \tau)^{1-\alpha} \sin \frac{\pi \alpha}{2}}{1+2(\omega \tau)^{1-\alpha} \sin \frac{\pi \alpha}{2}+(\omega \tau)^{2(1-\alpha)}} \\
\mu^{\prime \prime}=\left(\mu_{l}-\mu_{\infty}\right) \frac{(\omega \tau)^{1-\alpha} \cos \frac{\pi \alpha}{2}}{1+2(\omega \tau)^{1-\alpha} \sin \frac{\pi \alpha}{2}+(\omega \tau)^{2(1-\alpha)}}
\end{array}\right.
$$

This is the electromagnetic waves in inhomogeneous media, the magnetic energy loss factor $\mu$ " can be solved.

\section{Inhomogeneous medium quality factor}

Tunnel surrounding rock medium quality factor $\mathrm{Q}$ can be defined as the resonance frequency of the system, when the signal amplitude does not change over time, the system stored energy per cycle outside the energy ratio, that is deviation from the equilibrium point of the steady-state output exponential decay of the way, the incremental return to the steady-state output ${ }^{[6,7]}$.

$$
Q=2 \pi \frac{\text { EnergyStored }}{\text { EnergyDissipatedPerCycle }}
$$

Fig. 1 is cole-cole model equivalent circuit diagram, its microscopic simulation of rock conductivity, cole-cole model can calculate the amplitude and phase of the rock samples in the test band.

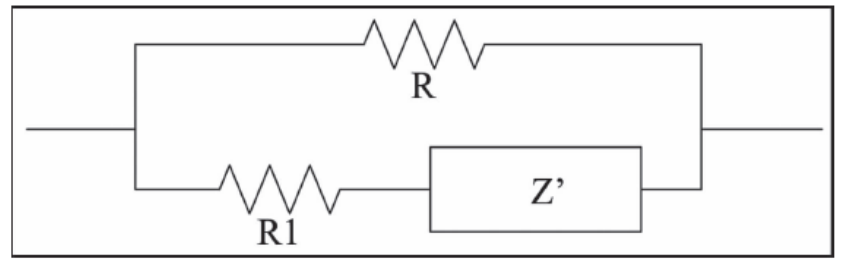

Fig. 1 Sketch of equivalent circuit of Cole-Cole model

$$
Z(i \omega)=z(0)\left\{1-m\left[1-\frac{1}{1+(i \omega \tau)^{c}}\right]\right\}
$$

Where: $z(0)$ is the frequency of 0 when the impedance; $m$ as the charging rate; $\tau$ for description of excitation polarization process of slow time constant, $c$ is the frequency correlation coefficient. $\omega$ is angular frequency of the alternating current field. The real and imaginary parts of decomposable:

$$
\left\{\begin{array}{l}
\operatorname{Re} Z(i \omega)=\frac{1-(1-m)(\omega \tau)^{2 c}+(2-m)(\omega \tau)^{c} \cos \frac{c \pi}{2}}{1+(\omega \tau)^{2 c}+2(\omega \tau)^{c} \cos \frac{c \pi}{2}} \\
\operatorname{LmZ}(i \omega)=\frac{-m(\omega \tau)^{c} \sin \frac{c \pi}{2}}{1+(\omega \tau)^{2 c}+2(\omega \tau)^{c} \cos \frac{c \pi}{2}}
\end{array}\right.
$$

The amplitude and phase of the rock samples in the test band:

$$
\begin{aligned}
& |Z|=\sqrt{[\operatorname{Re} Z(i \omega)]^{2}+[L m Z(i \omega)]^{2}} \\
& \theta=-\tan ^{-1} \frac{L m}{\operatorname{Re}}
\end{aligned}
$$

\section{The attenuation coefficient of radar electromagnetic wave propagation in inhomogeneous media}

According to the analysis of electromagnetic wave propagation in uniform media, the attenuation coefficient (19), the relaxation phenomena of electromagnetic waves in an inhomogeneous medium 
of electric energy and magnetic energy loss, as well as the non-uniform medium of tunnel surrounding rock quality factor of electrical energy and magnetic energy, compared various variables in the analysis (19) can be obtained for the attenuation coefficient of electromagnetic waves in the medium relaxation phenomena in inhomogeneous media:

$$
b(\omega)=\frac{\omega}{\varepsilon_{0}}\left\{\frac{1}{2} \frac{\mu_{l}(\omega)}{C_{0}} \operatorname{Re}\left[\varepsilon^{*}(\omega)\right] \sqrt{1+Q^{-1}(\omega)}-1\right\}
$$

\section{Conclusion}

Analysis of electromagnetic wave propagation in inhomogeneous media must consider the relaxation loss of the electrical and magnetic energy, while the tunnel surrounding the quality factor in determining the attenuation of the necessary ${ }^{[8]}$. These three parameters are not independent of unrelated, but from a different side to reflect the electromagnetic wave attenuation. Consider coupling between the three, Completed the solution of the attenuation coefficient of electromagnetic waves of radar waves in the non-uniform tunnel surrounding medium.

\section{Acknowledgements}

This work was financially supported by The National Natural Science Foundation of China (No. 50978129)

\section{References}

[1] Annan,A P. and Davis,J L.Impulse radar sounding in permafrost.Radio science II,1976,383 394

[2] Tong Xu,McMechen Geodge A.GPR attention and its numerical simulation in 2.5 dimensions. Geophysics[J],1997,62(1):403 414

[3] Von Hippel A R.Dielectrics and Waves[M].New York:John Wiley Sons,1962

[4] Tong M S,Ding Z.The Frequence Dispersion Models of Rock Complex Resistivity and Their Parameters Inversion[J].Well Logging Technology,2006,30(4),303 305

[5] Xiao Z S, Zeng Z G,Zhu S H,et al.An Experimental Study of Wettability Evaluation Based on Frequency Dispersion Property of Rock Electric Parameters[J],2009,52(5):1326 1332

[6] Igor Sevostianov ,Mark Kachanov.Explicit elasticity-conductivity connections for composites with anisotropic inhomogeneities[J].Mechanics and Physics of solids.2007,55:2181 2205

[7] Torqutao S.Modeling of physical properties of composite materials[J].Solids and Structures.2000, 37:411 422

[8] Pelton W H.Interpretation of induced polarization and resistivity data[D].Salt Lake City: University of Utah.1977 